\title{
Optical Möbius Strips in Three Dimensional Ellipse Fields: Lines of Linear Polarization
}

\author{
Isaac Freund \\ Department of Physics, and Jack and Pearl Resnick Advanced Technology Institute, \\ Bar-Ilan University, Ramat-Gan 52900, Israel
}

The minor axes of, and the normals to, the polarization ellipses that surround singular lines of linear polarization in three dimensional optical ellipse fields are shown to be organized into Möbius strips and into structures we call "rippled rings" (r-rings). The Möbius strips have two full twists, and can be either right- or left-handed. The major axes of the surrounding ellipses generate cone-like structures. Three orthogonal projections that give rise to 15 indices are used to characterize the different structures These indices, if independent, could generate 839,808 geometrically and topologically distinct lines; selection rules are presented that reduce the number of lines to 8,248 , some 5,562 of which have been observed in a computer simulation. Statistical probabilities are presented for the most important index combinations in random fields. It is argued that it is presently feasible to perform experimental measurements of the Möbius strips, r-rings, and cones described here theoretically. 


\section{INTRODUCTION}

We describe here the Möbius strips, and other structures, generated by the axes of the polarization ellipses that surround singular lines of linear polarization (L lines) in three dimensional (3D) optical ellipse fields. These strips have four half-twists; they complete the trinity of two-half-twist Mobius strips that surround ordinary ellipses $[1,2]$, and the one-half- and three-half-twist strips that surround singular lines of circular polarization (C lines) [3].

On an L line the polarization ellipse collapses to a line, the major axis of the ellipse, and the minor axis and ellipse normal become undefined (singular). All three axes - major, minor, and ellipse normal - remain well defined for the ellipses that surround the L line. In a plane $\Sigma$ pierced by an L line a point of linear polarization, an L point, appears. The projections onto $\Sigma$ of the minor axes and ellipse normals of the surrounding ellipses rotate about the point with winding number (net rotation angle divided by $2 \pi$ ) $I= \pm 1[4-13]$. Typical structures that surround L lines are shown in Fig. 1.

a
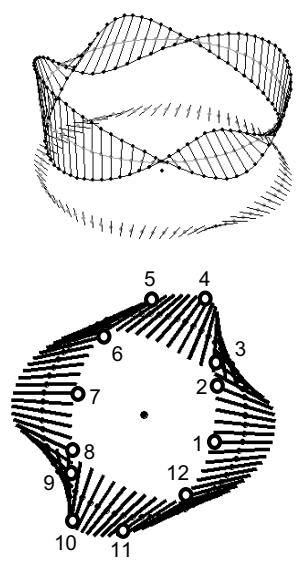

b

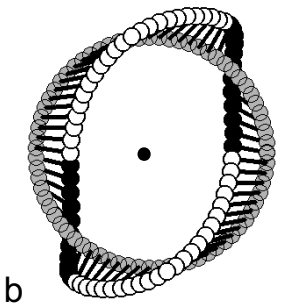

C
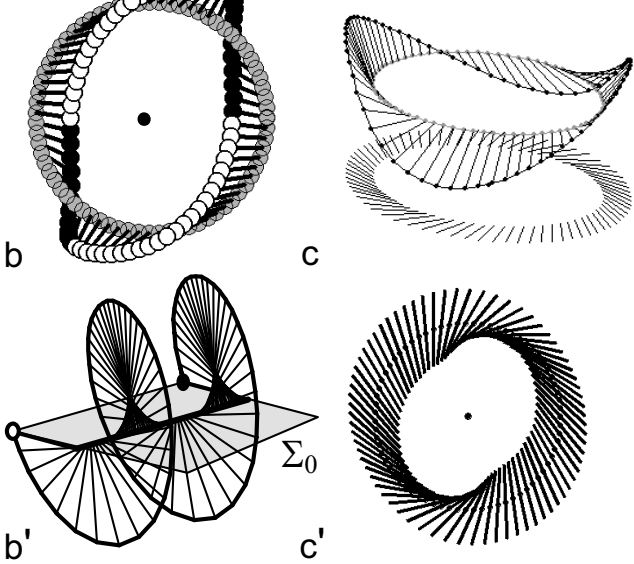

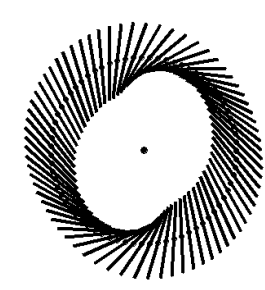

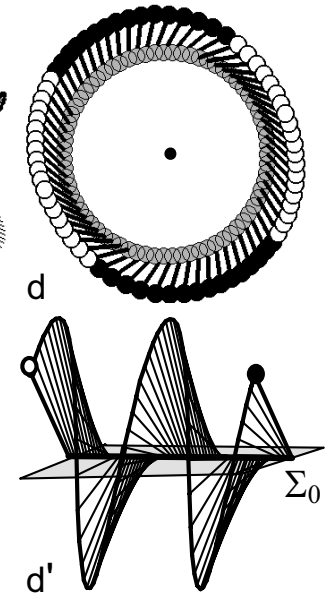

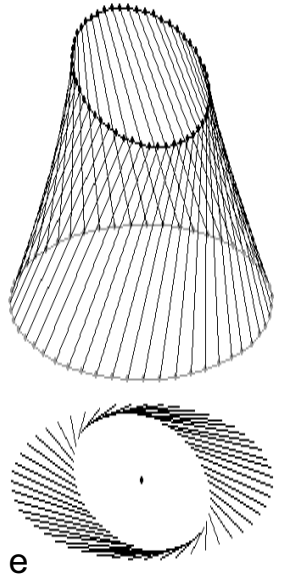

e

FIG. 1: Typical structures surrounding L lines in a (here computed) random 3D ellipse field. Shown in (a) - (d') (in (e)) are the structures generated by the minor (the major) axes of the ellipses whose centers are located in a plane $\Sigma_{0}$ on a small circle that surrounds the line. (a) 3D view of a Möbius strip that surrounds an L line shown floating above its projection onto $\Sigma_{0}$. (a') Enlarged view of the projections of the ellipse axes in (a) onto $\Sigma_{0}$. Along a counterclockwise path $(1-12)$ the axis projections rotate through $360^{\circ}$ in the retrograde, clockwise direction, and $I=-1$. Other Möbius strips have $I=+1$. (b) The strip in (a) viewed from above. For clarity, here and in (b'), (c), (d), and (d') only half of each axis is shown. Here and in (d) ellipse centers are shown by gray circles and their minor axes by short straight lines; axis endpoints that lie above (below) the plane of the circle of ellipse centers are shown by filled white (black) circles. As can be seen, ellipse endpoints and centers form a pair of interlocking rings with four crossings. (b') The closed Möbius strip in (a),(b) opened to better display the double twist structure. Here the ellipse centers form a straight line (the thick line) around which the axes of the surrounding ellipses (thin lines) rotate. The small white (black) circles mark the arbitrary first (last) ellipse. (c) 3D view of a second type of structure that surrounds L lines, shown floating above its projection onto $\Sigma_{0}$. This structure, which we call a rippled ring (r-ring), is not a Möbius strip. (c') Enlarged view of the axis projections onto $\Sigma_{0}$ of the r-ring in (c); the projections rotate through $360^{\circ}$ in the same direction as the path, and $I=+1$. Other r-rings have $I=-1$. (d) The r-ring in (c) viewed from above; the endpoints and centers form noninterlocking rings. (d') The closed r-ring in (c),(d) opened to display the ripple of the axes, which oscillate through two complete sinusoidal cycles. In a random field approximately $1 / 3$ of all $\mathrm{L}$ lines are surrounded by Möbius strips, $2 / 3$ by rippled rings. The normals to the surrounding ellipses (not shown) also generate Möbius strips and r-rings with $I= \pm 1$. (e) The major axes of the ellipses in $\Sigma_{0}$ generate cone-like structures, $\alpha$-cones, an example of which is shown floating above its projection onto $\Sigma_{0}$; here $I=+1$, other $\alpha$-cones have $I=-1$. 
Different types of $\mathrm{L}$ lines are characterized by the geometrical and topological indices of the structures that surround the lines. Topological index $I= \pm 1$ generates two distinct types of $\mathrm{L}$ lines $[4,7,11-13]$; the Mobius strips and other structures introduced here greatly expand the number of these lines. In later sections we use 15 different indices to characterize these structures; these 15 indices, if independent, could generate 839,808 different L lines. This number is drastically reduced to 8,248 by the selection rules described later; in a database containing $10^{6}$ independent realizations of a simulated random ellipse field we find some 5,562 different L lines. The true number of different lines is likely to be significantly greater, because as discussed later, due to the existence of highly improbable configurations the yield of different lines grows so slowly with the number of realizations that even $10^{9}$ independent realizations may be insufficient to generate all allowed possibilities.

The plan of this report is as follows. In Section II we describe our computer simulations and the numerical and analytical tools we use in later sections to study L lines, their Möbius strips, and related structures. In Section III we describe in detail the Möbius strips, r-rings, and $\alpha$-cones (Fig. 1) that surround L lines, and introduce 14 new indices to characterize these new structures. In Section IV we discuss the selection rules that constrain the number of possible index combinations, and present statistical probabilities for allowed combinations. We summarize our findings in the concluding Section V. Throughout, as in $[1,3]$, our approach is descriptive, a more mathematical treatment similar to [2] will be presented elsewhere.

\section{METHODS}

We briefly review here the methods we use to study $\mathrm{L}$ points on $\mathrm{L}$ lines. To a large extent these methods are the same as those used in [3] to study $\mathrm{C}$ points on $\mathrm{C}$ lines, and the reader may find it helpful to consult this reference for more details. Many of the concepts used here and in [3] have their origin in [1,2], and the reader may also find it helpful to consult these references.

The principal axis system for the general polarization ellipse is the (here right-handed) orthogonal three-frame $\boldsymbol{\alpha}, \boldsymbol{\beta}, \boldsymbol{\gamma}$, where $\boldsymbol{\alpha}$ and $\boldsymbol{\beta}$ are unit vectors directed along the major and minor ellipse axes, and $\gamma$ is a unit vector directed along the ellipse normal; the positive end of $\gamma$ can be uniquely defined by a right-hand rule based on the rotation of the electric field vector $\mathbf{E}$ as it traces out the ellipse over an optical cycle.

As noted above, at an $\mathrm{L}$ point on an $\mathrm{L}$ line there is only one principal axis, $\boldsymbol{\alpha}$, that is well defined. Normal to this axis is a plane, the principal plane here labelled $\Sigma_{0}$. In what follows, we consider the $3 \mathrm{D}$ structure of the ellipses whose centers lie in $\Sigma_{0}$ on a small circle $\sigma_{0}$ that surrounds the L point. We take the $z$-axis of the fixed, orthogonal coordinate system to be along $\boldsymbol{\alpha}$, and the corresponding $x y$-axes to lie in $\Sigma_{0}$, the orientation of these axes in this plane being arbitrary.

As the plane of observation $\Sigma$ is tilted relative to $\Sigma_{0}$ the $3 \mathrm{D}$ arrangement of the ellipses whose centers lie in $\Sigma$ changes. For very small tilt angles these changes affect only the geometries of the structures, but not their topologies, or their statistical properties. However, as the rotation angle increases past some small, critical value that differs for each L point, both the topology and the statistics change importantly. A similar phenomenon occurs for points of circular polarization, C points, on C lines [3]. All results presented in Fig. 11 (and unless stated otherwise in all other figures) are for the case $\Sigma=\Sigma_{0}$. The complex set of transformations that occur when $\Sigma$ is rotated away from $\Sigma_{0}$ will be reported on separately.

In general, $\boldsymbol{\alpha}$, and therefore $\Sigma_{0}$, make arbitrary angles with the L line itself, so that in moving along the line from one $\mathrm{L}$ point to another the coordinate system must be rotated in order to remain in the principal plane $\Sigma_{0}$ of each L point. 
$\boldsymbol{\alpha}, \boldsymbol{\beta}$, and $\boldsymbol{\gamma}$ can be calculated from the (here complex) optical field $\mathbf{E}$ using either

$$
\begin{aligned}
\boldsymbol{\alpha} & =\operatorname{Re}\left(\mathbf{E}^{*} \sqrt{\mathbf{E} \cdot \mathbf{E}}\right), \\
\boldsymbol{\beta} & =\operatorname{Im}\left(\mathbf{E}^{*} \sqrt{\mathbf{E} \cdot \mathbf{E}}\right), \\
\boldsymbol{\gamma} & =\operatorname{Im}\left(\mathbf{E}^{*} \times \mathbf{E}\right),
\end{aligned}
$$

which is due to Berry [11,13], or from the eigenvalues, $\lambda_{i}$, and eigenvectors, $\boldsymbol{\nu}_{i}, i=1,2$, 3 , of the $3 \times 3$ real coherency matrix [14] $M_{i j}=\operatorname{Re}\left(E_{i}^{*} E_{j}\right), i, j=x, y, z . \quad \gamma$ as defined in Eq. (1c) measures the area of the polarization ellipse and goes to zero at an L point. At an L point the polarization is linear and $\mathbf{E}$ can be made be pure real so that also $\boldsymbol{\beta}$ vanishes at the point. In what follows, $\boldsymbol{\alpha}$, $\boldsymbol{\beta}$, and $\boldsymbol{\gamma}$ for the surrounding ellipses are, without change in notation, always normalized to unit length, i.e. $\boldsymbol{\alpha} \Rightarrow \boldsymbol{\alpha} /|\boldsymbol{\alpha}|$, etc.

We study two computed 3D ellipse fields. The first is composed of a large number of linearly polarized plane waves with random propagation and polarization directions, and random phases [1]. This field is an exact solution of Maxwell's equations and serves as an important check on the structures found using the more convenient linear expansion described below. L lines in this field were traced out using the $\mathrm{L}$ point discriminant $D_{L}=a_{2}$ obtained from the characteristic equation $\lambda^{2}+a_{1} \lambda+a_{2}=0$ of $M_{i j}[15]$.

In the immediate vicinity of an L point the field describing the ellipses in $\Sigma_{0}$ can be expanded as

$$
\begin{aligned}
& E_{x}=\left(P_{x x}+\mathrm{i} Q_{x x}\right) x+\left(P_{x y}+\mathrm{i} Q_{x y}\right) y, \\
& E_{y}=\left(P_{y x}+\mathrm{i} Q_{y x}\right) x+\left(P_{y y}+\mathrm{i} Q_{y y}\right) y, \\
& E_{z}=a+\left(P_{z x}+\mathrm{i} Q_{z x}\right) x+\left(P_{z y}+\mathrm{i} Q_{z y}\right) y,
\end{aligned}
$$

where the direction of polarization of the point is along the $z$-axis and $\Sigma_{0}$ is the $x y$-plane.

In many cases simpler expansions suffice: the Möbius strip in Fig. 1a,b is closely approximated by the field model $E_{x}=i x+(1-i) y, E_{y}=-i y, E_{z}=-1$, the r-ring in Fig. 11e,d by $E_{x}=$ $-(1+i) x, E_{y}=i x+(1-i) y, E_{z}=1$, and the $\alpha$-cone in Fig. 11p by $E_{x}=-x, E_{y}=x-y, E_{z}=1$.

The statistics in $\Sigma_{0}$ of $a$ and the $P, Q$ in Eq. (2) were obtained using the numerical procedure described in [3] for $\mathrm{C}$ points, modified for $\mathrm{L}$ points by the fact that whereas $\mathbf{E} \cdot \mathbf{E}=0$ for a $\mathrm{C}$ point, $\mathbf{E}^{*} \times \mathbf{E}=0$ for an L point $[11-13]$. The probability density functions (PDFs) in $\Sigma_{0}$ of $a$, and of $P, Q$ are shown in Fig. 2 - here all $P, Q$ have the same PDF.
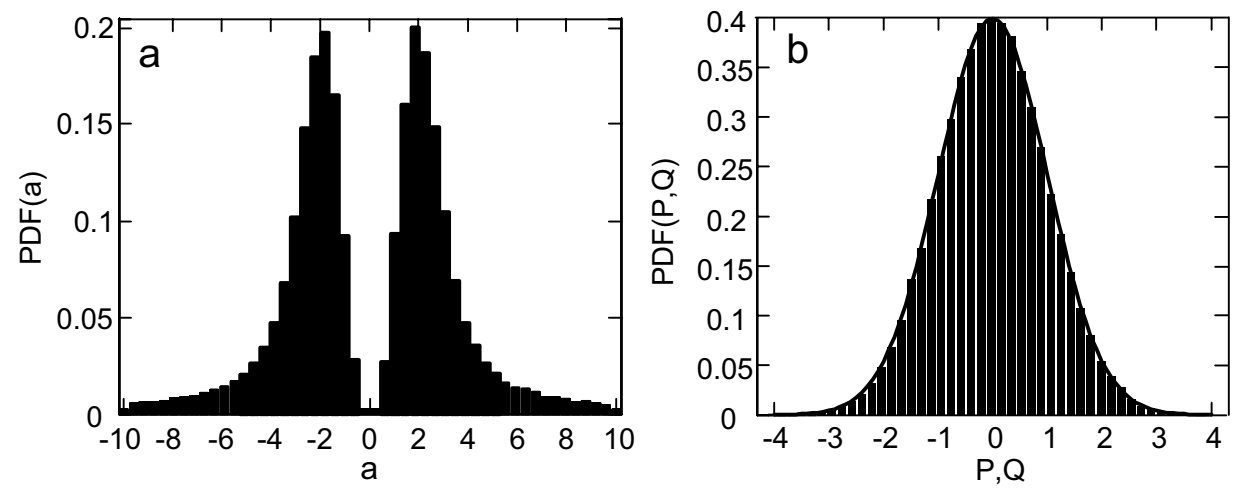

FIG. 2: PDFs in $\Sigma_{0}$ of the parameters $a, P$, and $Q$, in Eq. (2). (a) PDF of $a$. (b) PDF of $P, Q$. The numerical data (histogram) closely matches a Gaussian with unit variance (curve). 
A single projection, the $\Sigma_{0}$ projection, cannot, of course, fully describe the complex $3 \mathrm{D}$ arrangement of the ellipses surrounding an L line, and in addition to projecting the ellipses on $\sigma_{0}$ onto $\Sigma_{0}$ we study two additional projections $-\tau_{0}$ and $\pi_{0}$. These projections, which are the same as those used in $[1-3]$, are reviewed in Fig. 3. Thus, in characterizing the 3D arrangement of the ellipses in the vicinity of an $\mathrm{L}$ line we use three orthogonal projections, $\Sigma_{0}, \tau_{0}$, and $\pi_{0}-$ the minimum number required for a complex object.

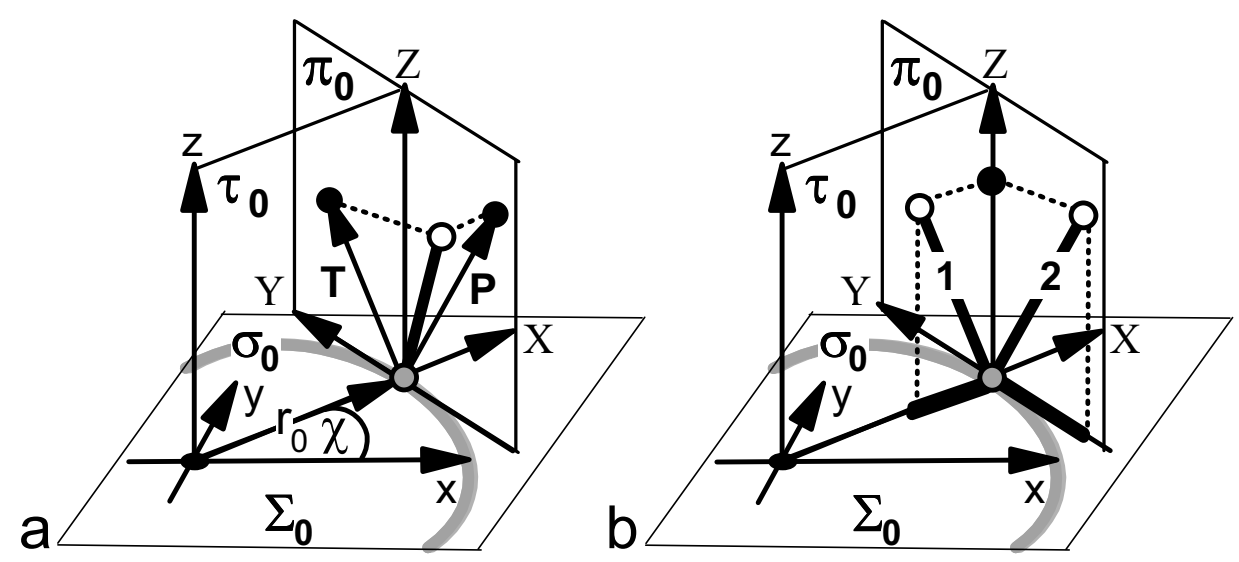

FIG. 3: Projections $\tau_{0}$ and $\pi_{0}$. The L point is at the origin of the fixed $x y z$-coordinate system. (a) Rotating plane $\tau_{0}$, the $X Z$-plane $\left(\pi_{0}\right.$, the $Y Z$-plane), is radial (tangential) to $\sigma_{0}$ and travels on $\sigma_{0}$, rotating about the fixed $z$-axis; its position on $\sigma_{0}$ is measured by the angle $\chi$, and by arc length $s=r_{0} \chi$. An axis, $\boldsymbol{\alpha}$, $\boldsymbol{\beta}$, or $\boldsymbol{\gamma}$, of the ellipse whose center (small gray circle) is located at the origin of $X Y Z$ is projected onto $\tau_{0}$ $\left(\pi_{0}\right)$. The endpoint of the axis is shown by a small white circle, the axis itself by a thick line connecting the center and the endpoint. The endpoint projection in $\tau_{0}\left(\right.$ in $\left.\pi_{0}\right)$ is shown by a small black circle. The vector $\mathbf{T}(\mathbf{P})$ in $\tau_{0}$ (in $\pi_{0}$ ) connects the ellipse center with the endpoint projection. As $\tau_{0}\left(\pi_{0}\right)$ moves along $\sigma_{0}$ different ellipses are projected onto $\tau_{0}\left(\pi_{0}\right)$, their endpoints in $\tau_{0}\left(\pi_{0}\right)$ trace out a curve, the $\tau_{0}\left(\pi_{0}\right)$ endpoint curve, and vector $\mathbf{T}(\mathbf{P})$ rotates. As discussed in Section III, the winding numbers of rotation of $\mathbf{T}(\mathbf{P})$ and of the tangent to the endpoint curve in $\tau_{0}$ (in $\pi_{0}$ ), give rise to indices $\tau_{i}$ and $d \tau_{i}\left(\pi_{i}\right.$ and $\left.d \pi_{i}\right), i=\alpha, \beta, \gamma$, that characterize the 3D arrangement of the ellipses on $\sigma_{0}$. $\quad$ (b) Special projections. When the endpoint projection onto $\tau_{0}$ (onto $\pi_{0}$ ) of an ellipse axis lies on the $Z$-axis, as shown here for an axis of ellipse 1 (ellipse 2 ), the axis projection onto $\Sigma_{0}$ is radial to $\sigma_{0}$, i.e. along the $X$-axis (is tangential to $\sigma_{0}$, i.e. along the $Y$-axis). These two important special cases are elaborated on in Section III.

Issues regarding scaling and other technical aspects of the L point graphics presented here are the same as those for the $\mathrm{C}$ point graphics presented in [3]; the reader will find a full discussion of these issues in [3].

Unless stated otherwise, throughout we use a single value for the radius $r_{0}=10^{-4}$ of the surrounding circle $\sigma_{0}$. This value is sufficiently small that except for a scale factor the wavefield structure is invariant under further reduction of the radius. The fact that every small value for $r_{0}$ yields the same structure implies that the L point is surrounded by nested Möbius strips, r-rings, $\alpha$-cones, etc. The full 3D arrangements of these deeply nested structures are, of course, quite impossible to visualize; accordingly, below we dissect out a single, typical surrounding circle, the $\sigma_{0}$ circle with $r_{0}=10^{-4}$, and proceed to study its properties in detail. 


\section{INDICES OF MÖBIUS STRIPS, R-RINGS, AND $\alpha$-CONES}

Here we discuss the 12 winding numbers that describe the Möbius strips, r-rings, and $\alpha$-cones that surround L lines, as well as the line classification [16] and its relationship to these indices.

\section{A. Indices of the Projection onto $\Sigma_{0}: I_{\beta, \gamma}$ and $I_{\alpha}$}

As a result of the studies of Nye and coworkers [4-10], it has long been known that the winding number of the projection of axis $\boldsymbol{\beta}$ onto $\Sigma_{0}, I_{\beta}= \pm 1$ (Figs. 1 $1 \mathrm{a}^{\prime}, \mathrm{c}^{\prime}$ ), equals $I_{\gamma}$, the corresponding winding number of axis $\gamma$. The reason for the equivalence of these two indices is that as the radius of the surrounding circle shrinks towards zero, by continuity the major axes of the ellipses on this circle all approach parallelism with the direction of polarization of the $\mathrm{L}$ point. The projections of $\boldsymbol{\beta}$ and $\boldsymbol{\gamma}$ onto $\Sigma_{0}$ are therefore substantially orthogonal, and as one rotates so does the other. Accordingly, in what follows we use the symbol $I_{\beta, \gamma}$ to denote the common winding number of these two axes. Although perhaps less obvious, the equivalence $I_{\beta}=I_{\gamma}$ holds also for arbitrary $\Sigma$ [13].

The winding number $I_{\alpha}$ of the projection of axis $\boldsymbol{\alpha}$ onto $\Sigma_{0}$ does not appear to have been discussed previously. We find that just like for $I_{\beta, \gamma}$, in all cases $I_{\alpha}= \pm 1$ (Fig. 1p).

$I_{\alpha}$ and $I_{\beta, \gamma}$ are independent, and we find all four possible sign combinations in our simulations.

It is not difficult to derive analytical expressions for the above indices. Writing $\mathbf{V}$ for axis $\boldsymbol{\alpha}$, $\boldsymbol{\beta}$, or $\boldsymbol{\gamma}$, we have from Eqs. (1) and (2) for the $x y$-components of $\mathbf{V}$ for sufficiently small $x, y$,

$$
\begin{aligned}
& V_{x}=F_{x x} x+F_{x y} y, \\
& V_{y}=F_{y x} x+F_{y y} y,
\end{aligned}
$$

where for

$$
\begin{array}{ll}
\operatorname{axis} \boldsymbol{\alpha}: & F=P \text { (i.e. } F_{x x}=P_{x x} \text {, etc.); } \\
\text { axis } \boldsymbol{\beta}: & F=Q ; \\
\text { axis } \boldsymbol{\gamma}: & F_{x x}=-2 a Q_{y x}, F_{x y}=-2 a Q_{y y}, F_{y x}=2 a Q_{x x}, F_{y y}=2 a Q_{x y} .
\end{array}
$$

Berry [13] has given the formula $I=\operatorname{sign}\left(F_{x x} F_{y y}-F_{x y} F_{y x}\right)$ for a general vector field of the form in Eq. (3). Inserting Eq. (4) into Berry's formula, we have

$$
\begin{aligned}
& I_{\alpha}=\operatorname{sign}\left(P_{x x} P_{y y}-P_{x y} P_{y x}\right), \\
& I_{\beta}=I_{\gamma}=I_{\beta, \gamma}=\operatorname{sign}\left(Q_{x x} Q_{y y}-Q_{x y} Q_{y x}\right) .
\end{aligned}
$$

We find this result to be in full agreement with the indices obtained numerically. 


\section{B. Indices of the Projections onto $\tau_{0}$ and $\pi_{0}$}

\section{Projections onto $\tau_{0}$}

a. Indices $\tau_{i}$ and $d \tau_{i}, i=\beta, \gamma$

Möbius strips Both axes $\boldsymbol{\beta}$ and $\boldsymbol{\gamma}$ of the surrounding ellipses can generate Möbius strips that are either right- or left-handed screws with two full turns.

Index $\tau_{i}$ measures the number of turns and their handedness $(\operatorname{sign})$, and is $\tau_{i}=+2\left(\tau_{i}=-2\right)$ for a left-handed (right-handed) Möbius strip. As can be seen, the Möbius strip in Fig. 1 1 a-b' is a left handed screw with $\tau_{\beta}=+2$.

Although not an obvious geometrical or topological necessity, we find in $\Sigma_{0}$ that if $\tau_{\beta}=+2$ $\left(\tau_{\beta}=-2\right)$ then $\tau_{\gamma} \neq-2\left(\tau_{\gamma} \neq+2\right)$, and vice versa. If both axes $\boldsymbol{\beta}$ and $\boldsymbol{\gamma}$ generate Mobius strips (which need not, and does not, always occur), then the above rule implies that both strips must have the same handedness.

Index $d \tau$ measures the rotation of the tangent to the endpoint curve. For Möbius strips, and only for Möbius strips, we find that in all cases $d \tau_{i}=\tau_{i}$, i.e. when $\tau_{i}=+2\left(\tau_{i}=-2\right) d \tau_{i}=+2$ $\left(d \tau_{i}=-2\right)$. Because $\tau_{\beta}=\tau_{\gamma}$ when both axes generate Möbius strips, $d \tau_{i}=\tau_{i}$ implies that for such paired strips $d \tau_{\beta}=d \tau_{\gamma}$.

Indices $\tau_{i}$ and $d \tau_{i}$ for L point Möbius strips are illustrated in Fig. 4.
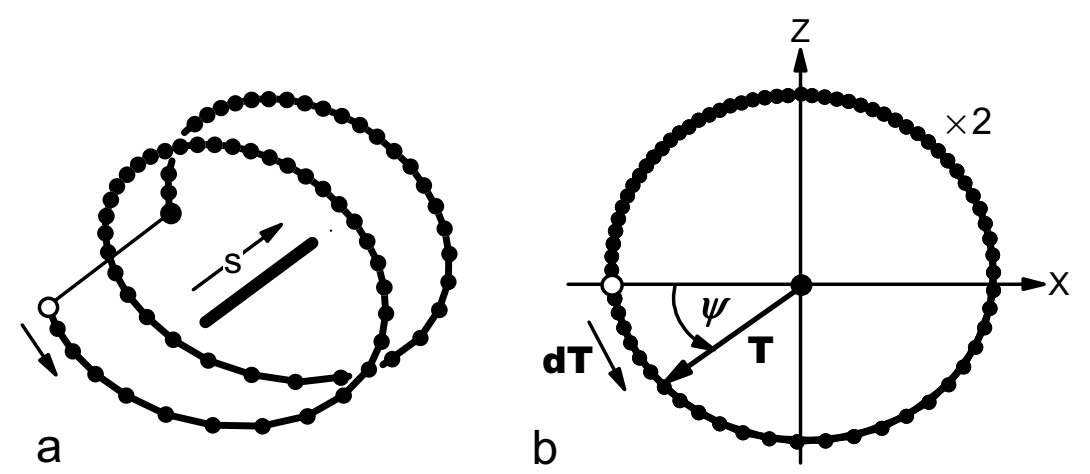

FIG. 4: Möbius strip endpoint curves in $\tau_{0}$. Here and throughout, small, connected black circles show the curve generated by the projections of axis endpoints onto $\tau_{0}$ as $\tau_{0}$ rotates on $\sigma_{0}$ through one complete circuit around the L point; the starting point of the circuit is shown by a small white circle; the sequence in which the endpoint curve is drawn is indicated by a small arrow. (a),(b) $\tau_{0}$ endpoint curve of the Möbius strip in Figs. 1 1 -b'. (a) 3D view of the endpoint curve. The first and last points are shown connected because they are the same point. The central straight line is formed by the centers of the ellipses on $\sigma_{0}$. As can be seen, the endpoints generate a two-turn left-handed helix. (b) The endpoint curve in (a) viewed normal to $\tau_{0}$. The " $\times 2$ " label indicates that the second turn of the helix in (a) lies behind the first turn visible here. Vector $\mathbf{T}$ has its origin at the center of the ellipse on the surrounding circle $\sigma_{0}$ (the origin of $X Z$ ) and its head at the endpoint projection. The winding number $\tau_{i}$, where for the Möbius strip shown here $i=\beta$, is obtained from the rotation of $\mathbf{T}$ by measuring the rotation angle $\psi(s)=\arctan \left(T_{Z}(s), T_{X}(s)\right)$, and unwrapping (unfolding) the result to obtain $\tau_{i}=[\psi(2 \pi)-\psi(0)] /(2 \pi)$. The winding number $d \tau_{i}$ of the tangent vector to the endpoint curve, $\mathbf{d T}$, is similarly calculated from winding angle $\arctan \left(d T_{Z} / d s, d T_{X} / d s\right)$. Here the net total rotation of $\mathbf{T}$ and of $\mathbf{d T}$ is $+4 \pi$, and $\tau_{i}=d \tau_{i}=+2$. Most endpoint curves do not have a symmetrical, near circular shape, as does the one shown here, which was chosen for simplicity and clarity of presentation. Although here $\tau_{i}=d \tau_{i}$, in general these two indices can be different. 
An important property of L point Möbius strips is that the first and second turn of the two-turn helix are identical, so that the projections onto $\tau_{0}$ of the two turns always overlap and only one turn is seen in such a projection, Fig. 4. We therefore include in such projections a " $\times 2$ " label to alert the reader to the fact that there is a second turn underlying the first.

r-rings The endpoint curves of r-rings form two-turn helices - possibly surprising because such a structure cannot be easily inferred from Figs. 1 $1 c-d$ '. For r-rings, $\tau_{i} \equiv 0$, whereas $d \tau_{i}=$ $0, \pm 2$. Typical examples are shown in Fig. 5, which also illustrates the "phase ratchet rules" that simplify calculation of $\tau_{i}$ for complicated endpoint curves [3]. For paired $\boldsymbol{\beta}-\boldsymbol{\gamma}$ r-rings $d \tau_{\beta}$ and $d \tau_{\gamma}$ need no longer be equal, and we find in our simulations all nine possible combinations of these two indices.

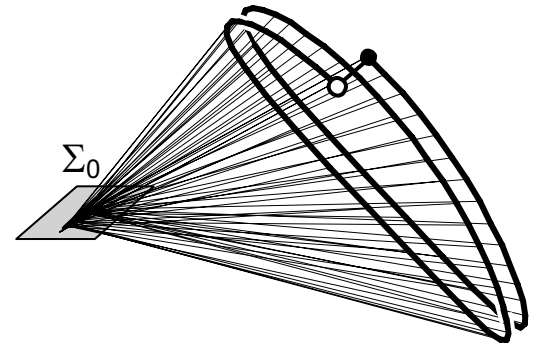

a

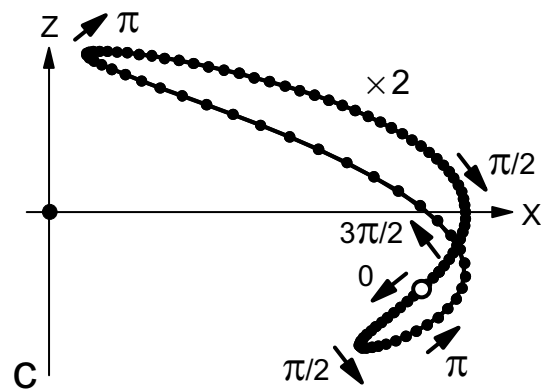

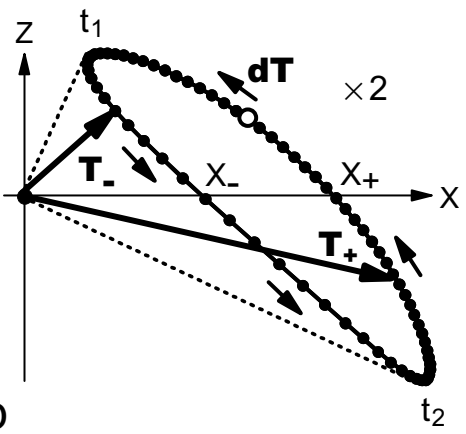

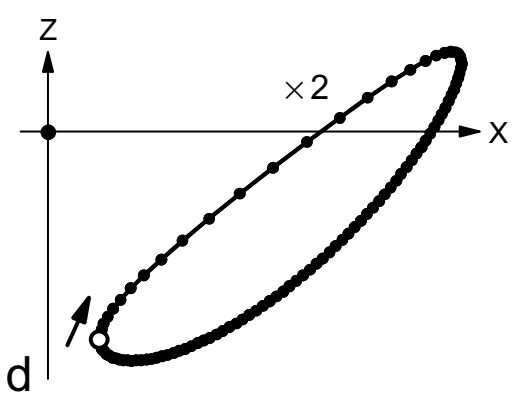

FIG. 5: r-ring endpoint curves in $\tau_{0}$. (a) The opened r-ring in Fig. 11 d' viewed from the left to display the two-turn helix generated by the axis endpoints. (b) Endpoint curve of (a). Because the endpoints of an r-ring do not spiral around the line of ellipse centers, the origin of the vector $\mathbf{T}$ always lies outside the closed endpoint curve, and as a result $\tau_{i} \equiv 0$. This can be seen by following the rotation of $\mathbf{T}$ or by using the phase ratchet rules. At turning points $t_{1}$ and $t_{2}$ vector $\mathbf{T}$ reverses its direction of rotation, which is counterclockwise, i.e. positive, (clockwise, i.e. negative) on the right (left) half of the endpoint curve, and the net rotation of $\mathbf{T}$ is zero. In applying the phase ratchet rules [3], one first lists the signed crossing of the endpoint curve with the $X Z$-axes in the order that they occur, starting at the small white circle that marks the beginning of the circuit on $\sigma_{0}$ and proceeding in the direction of the small arrow. For the endpoint curve of the Möbius strip in Fig. 4 4 the crossing sequence is $Z_{+} X_{+} Z_{+} X_{+}$, where the sign of a crossing is positive (negative) if $\mathbf{T}$ rotates counterclockwise (clockwise) at the crossing. This is the canonical sequence for $\tau_{i}=+1$; multiplying by 2 to account for the hidden second turn yields the net index $\tau_{i}=+2$. For the r-ring shown here the crossing sequence is $X_{-} X_{+}$. Adjacent terms in the same axis necessarily have opposite signs and cancel, so for this sequence $\tau_{i}=0$. The tangent vector, $\mathbf{d T}$, however, is easily seen to rotate through $2 \pi$ in the positive, clockwise direction; multiplying by 2 , the net winding angle is $4 \pi$ and $d \tau_{i}=+2$. (c) An r-ring whose endpoint curve forms a figure of eight. Here the crossing sequence is $X_{+} X_{-}$ and once again $\tau_{i}=0$, as it is for every r-ring. But here also $d \tau_{i}=0$, as it is for every figure of eight. That the net rotation of $\mathbf{d T}$ is zero can be seen by following the tangent vectors marked with the cumulative rotation angle. (d) An r-ring with $\tau_{i}=0$ and $d \tau_{i}=-2$. In a random field there are roughly equal numbers of each type of r-ring: $\tau_{i}=0$ and $d \tau_{i}=-2,0,+2$. 
b. Index $d \tau_{\alpha}$ As illustrated in Fig. 17, axes $\boldsymbol{\alpha}$ of the surrounding ellipses are not organized into Möbius strips or r-rings, but rather into cone-like structures $-\alpha$-cones. In Fig. 6 we show additional views of the cone in Fig. 11. As can be seen, the endpoint curve of an $\alpha$-cone is a twoturn helix, much like the helix of an r-ring, and like an r-ring, $\tau_{\alpha} \equiv 0$ also for the cone; once again the reason is that the endpoint curve does not encircle the ellipse centers and vector $\mathbf{T}$ therefore oscillates clockwise and counterclockwise with a net winding angle of zero. Unlike r-rings, however, the endpoint curves of $\alpha$-cones are never figures of eight, and for the cones $d \tau_{\alpha}= \pm 2$.

For the $\alpha$-cone in Fig. 6a the axis endpoints form an ellipse that is smaller than the circle of ellipse centers (i.e. the surrounding circle). But for other cones, or for this cone viewed from below rather than from above, the endpoint ellipse is larger than the circle. In either case the two curves - circle and ellipse - never cross. There are, however, numerous examples of cones where the major axis of the endpoint ellipse is larger than, and the minor axis is smaller than, the diameter of the circle of ellipse centers, and in these cases the two curves - circle and ellipse - cross four times.
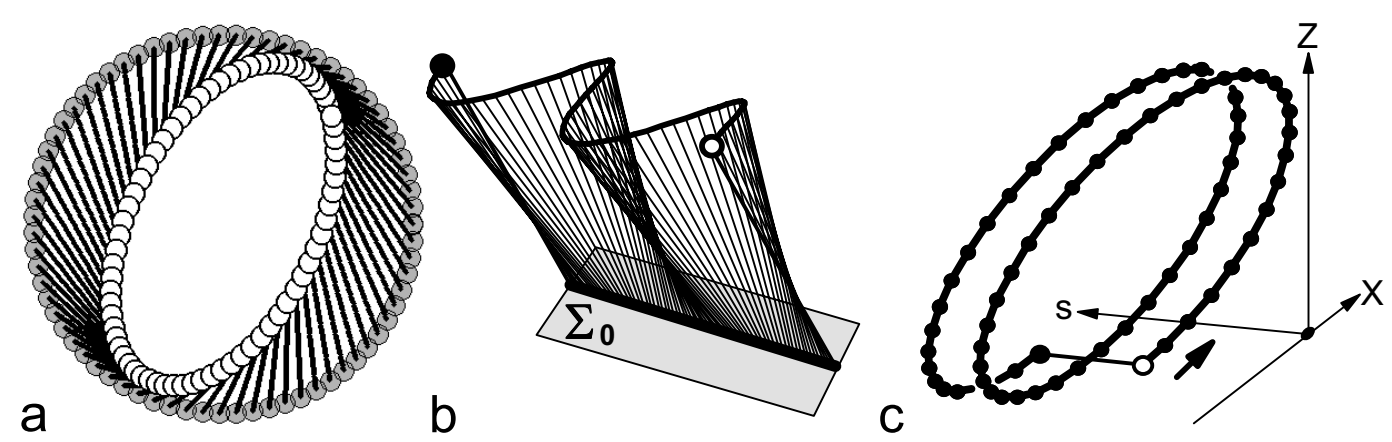

FIG. 6: $\alpha$-cones. Shown are three additional views of the cone in Fig. 1. (a) The cone viewed from above. (b) The cone in (a) opened and straightened to show the oscillations of the axis endpoints. (c) The $\tau_{0}$ projection of the axis endpoints. Here the $Z$-axis is elongated relative to the $X$-axis to show the structure of the normally very flat two-turn endpoint helix that is characteristic of $\alpha$-cones.

\section{Projections onto $\pi_{0}$}

a. Indices $\pi_{i}$ and $d \pi_{i}, i=\beta, \gamma \quad$ Indices $\pi_{i}$ and $d \pi_{i}$ are the $\pi_{0}$ projection analogs of indices $\tau_{i}$ and $d \tau_{i}$ of the $\tau_{0}$ projection.

Index $\pi_{i}$ measures the rotation of the endpoint curve in $\pi_{0}$, whereas index $d \pi_{i}$ measures the rotation of the tangent to this curve. Fig. 4 is applicable to $\pi_{i}$ and $d \pi_{i}$ upon replacement of the $X$-axis by the $Y$-axis, and vectors $\mathbf{T}$ and $\mathbf{d T}$ by vectors $\mathbf{P}$ and $\mathbf{d P} . \quad \pi_{i}$ and $d \pi_{i}$ can each take on the values $0, \pm 2$.

$\left|\tau_{\beta}\right|=2\left(\left|\tau_{\gamma}\right|=2\right)$ implies that axis $\boldsymbol{\beta}$ (axis $\gamma$ ) generates a Mobius strip, because for $\mathrm{r}$-rings (and cones) $\tau_{i} \equiv 0$. But the same is not true for $\pi_{i}$ which equals \pm 2 if the endpoint curve in $\pi_{0}$ encloses the ellipse centers, and is zero otherwise. In very nearly $1 / 4$ of all r-rings $\left|\pi_{i}\right|=2$.

There exists a $\pi_{i}$ selection rule that is the analog of the $\tau_{i}$ selection rule noted above: when $\pi_{\beta}=+2\left(\pi_{\beta}=-2\right), \pi_{\gamma} \neq-2\left(\pi_{\gamma} \neq+2\right)$.

$d \pi_{i}$ is \pm 2 for simple (non-self-intersecting curves), and is zero for figures of eight. When $\pi_{i}=+2$ $\left(\pi_{i}=-2\right), d \pi_{i}=+2\left(d \pi_{i}=-2\right)$.

Fig. 7 illustrates the case of an r-ring with $\tau_{\beta}=d \tau_{\beta}=0$, and $\pi_{\gamma}=d \pi_{\gamma}=-2$. 

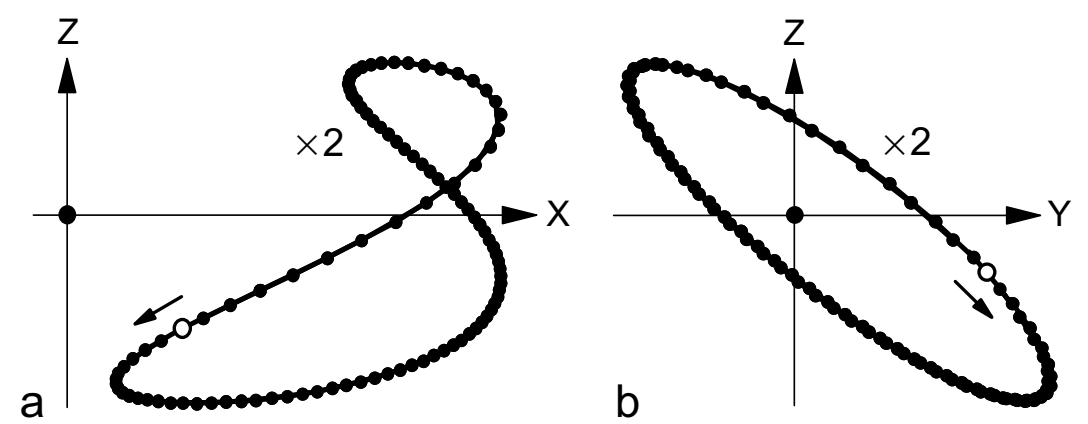

FIG. 7: r-ring endpoint curves of axis $\gamma$ in $\tau_{0}$ and in $\pi_{0}$. (a) $\tau_{0}$ endpoint curve. Both $\tau_{\gamma}$ and $d \tau_{\gamma}$ are zero, as is always the case for a figure of eight. (b) $\pi_{0}$ endpoint curve. The curve encloses the origin (circle of ellipse centers), and both $\pi_{\gamma}$ and $d \pi_{\gamma}$ equal -2 .

b. Index $d \pi_{\alpha}$ Indices $\pi_{\alpha}$ and $d \pi_{\alpha}$ are the $\pi_{0}$ projection analogs of indices $\tau_{\alpha}$ and $d \tau_{\alpha}$ of the $\tau_{0}$ projection. Like the endpoint curve of axis $\boldsymbol{\alpha}$ in $\tau_{0}$, for which $\tau_{\alpha} \equiv 0$ and $d \tau_{\alpha}= \pm 2$, the endpoint curve in $\pi_{0}$ generates a two-turn helix for which $\pi_{\alpha} \equiv 0$ and $d \pi_{\alpha}= \pm 2$. Fig. 65 for the $\tau_{0}$ projection is applicable also to the $\pi_{0}$ projection after the $X$-axis is replaced by the $Y$-axis.

This completes our discussion of the 12 indices that characterize the three orthogonal projections, $\Sigma_{0}, \tau_{0}$, and $\pi_{0}$, of the Mobius strips, r-rings and $\alpha$-cones that surround $\mathrm{L}$ lines. In the next section we return to the $\Sigma_{0}$ projection and consider a characterization [16] that is well known for $\mathrm{C}$ lines $[4,6,11-13]$, but that does not appear to have been discussed previously for $\mathrm{L}$ lines.

\section{Line Classification: Indices $\Lambda_{i}, i=\alpha, \beta, \gamma$}

\section{Computer Simulation}

The arrangement of the axis projections onto $\Sigma_{0}$ can be characterized by two indices. The first, winding numbers $I_{\alpha}$ and $I_{\beta}=I_{\gamma}=I_{\beta, \gamma}$, measures the net rotation of the axis projections around the L point, and has been discussed in Section III.A and illustrated in Fig. 1. The second, the line classification index $\Lambda_{i}, i=\alpha, \beta, \gamma$, counts the number of streamlines formed by axis $i$ that terminate (or originate) on the $\mathrm{L}$ point as straight lines (separatixes). For a sufficiently small surrounding circle $\sigma_{0}$, as is used here, $\Lambda_{i}$ counts the number of axis projections on $\sigma_{0}$ that point directly at the $\mathrm{L}$ point. We find that in all cases, for positive (negative) $I_{i}, \Lambda_{i}=0,4$ (4). We note that although the line classification [16] has not been previously discussed explicitly for $\mathrm{L}$ points for any axis, these values are consistent with streamline maps for axes $\boldsymbol{\beta}$ and $\boldsymbol{\gamma}$ presented in $[4,7,11]$; streamlines for axis $\boldsymbol{\alpha}$ do not appear to have been considered previously.

Unlike the case of winding number $I$ which is always the same for both axes $\boldsymbol{\beta}$ and $\boldsymbol{\gamma}, \Lambda_{\beta}$ and $\Lambda_{\gamma}$ can differ when $I_{\beta, \gamma}=+1$. Examples of the line classification are illustrated in Fig. 8 . An important, general property of these maps is that they posses a center of inversion. This follows from the forms of Eqs. (1) and (2) for sufficiently small $x, y$. But this is the only intrinsic symmetry of such maps, and therefore of the corresponding streamlines.

As indicated in Fig. 3, an axis projection points towards the L point (circle center) only when the axis itself lies in the $X Z$-plane, so that the projection of its endpoint onto $\pi_{0}$ lies on the $Z$-axis. Also this property is illustrated in Fig. 8. Thus, $\Lambda$ also equals the number of times the endpoint curve in $\pi_{0}$ crosses the $Z$-axis, and vice versa. 

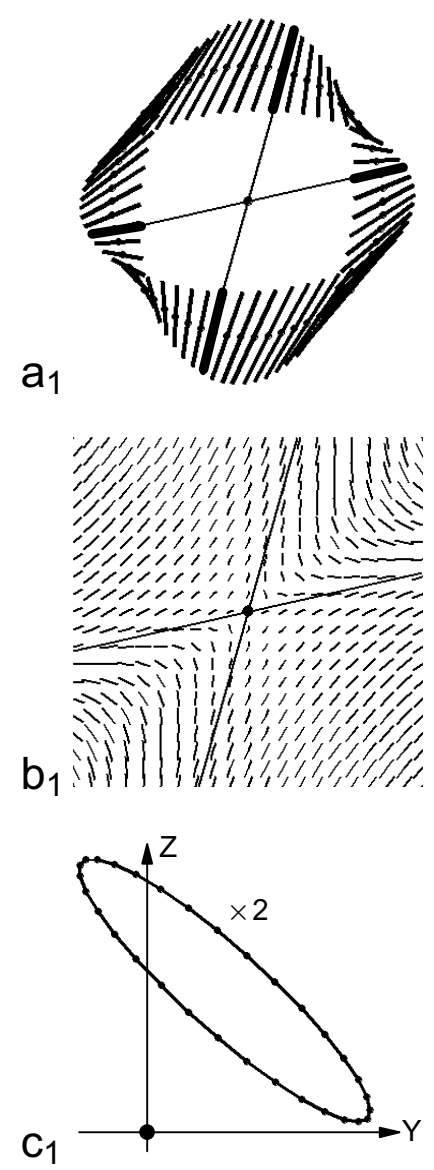

$a_{2}$
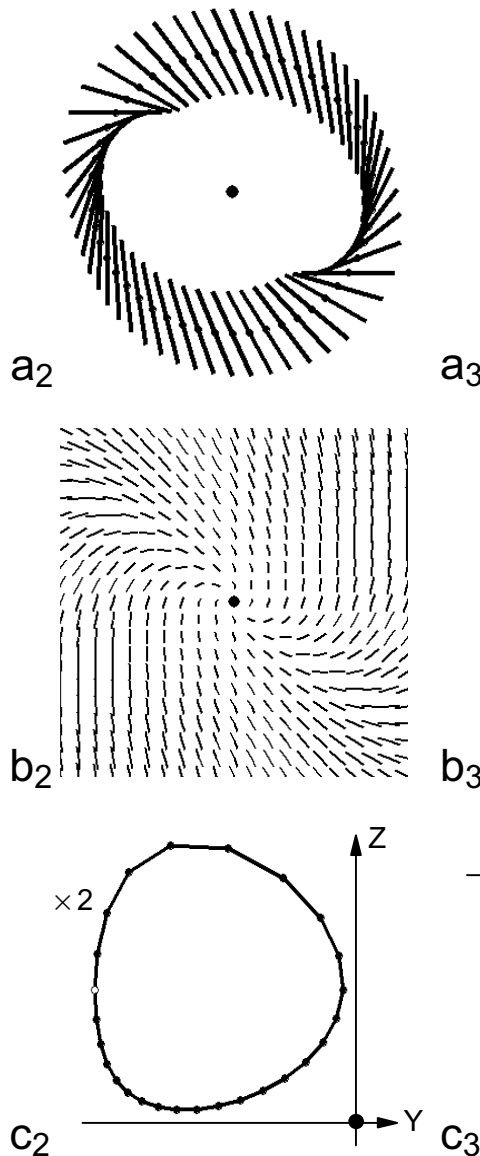

$a_{3}$
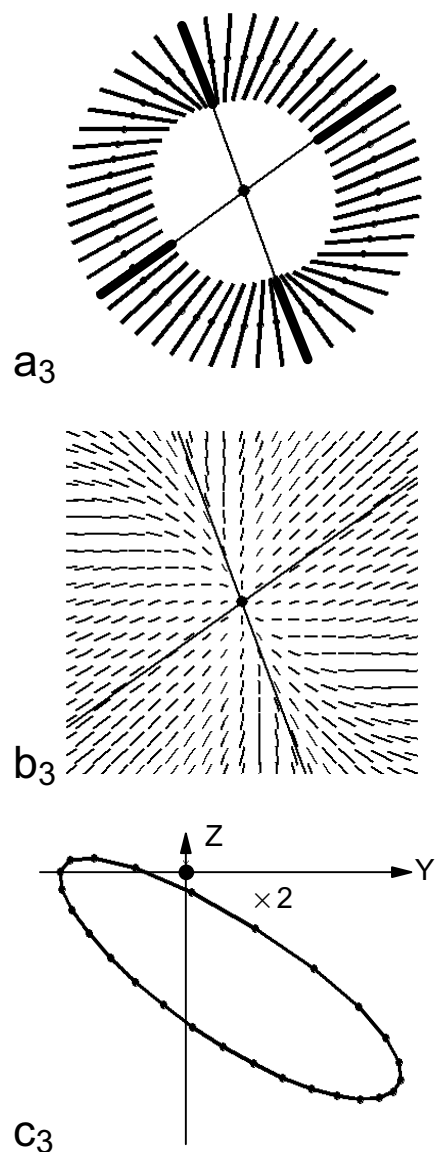

FIG. 8: Line classification in a computed 3D random ellipse field. $\left(\mathrm{a}_{1}\right)-\left(\mathrm{a}_{3}\right)$ Axis projections onto $\Sigma_{0}$. Shown as short straight lines are the projections of the axes of the ellipses on the surrounding circle $\sigma_{0}$. Projections that point directly at the circle center (L point), here $\Lambda$-lines, are marked by thick lines; the line classification index $\Lambda$ counts the number of these lines. In our simulation $\Lambda$-lines are found numerically [3] as the solutions of $\theta(\chi)=\chi+n \pi$, with $n=-4 \ldots 4$, as required. Here $\theta$ is the angle that the axis projection makes with the $x$-axis, and $\chi$ measures the position of the ellipse center on $\sigma_{0}$, Fig. 3 . The straight lines passing through the L point are calculated from Eq. 99. As can be seen, in each case shown here, and in numerous other cases tested but not shown, there is complete agreement between theory and the simulated field. ( $\left.\mathrm{a}_{1}\right)$ Axis $\boldsymbol{\alpha}$. Here index $I_{\alpha}=-1, \Lambda_{\alpha}=4$; for other $\alpha$-cones $I_{\alpha}=+1, \Lambda_{\alpha}=0,4$. $\left(\mathrm{a}_{2}\right)$ Axis $\boldsymbol{\beta}$. Here $I_{\beta}=+1, \Lambda_{\beta}=0$; for other $\boldsymbol{\beta}$ Möbius strips or r-rings $I_{\beta}=-1,+1, \Lambda_{\beta}=4 . \quad\left(\mathrm{a}_{3}\right)$ Axis $\gamma$. Here $I_{\gamma}=+1, \Lambda_{\gamma}=4$; for other $\gamma$ Möbius strips or r-rings $I_{\gamma}=-1, \Lambda_{\gamma}=4$, or $I_{\gamma}=+1$, $\Lambda_{\gamma}=0 . \quad\left(b_{1}\right)-\left(b_{3}\right)$ Axis projections onto $\Sigma_{0}$ corresponding to the ellipse fields in $\left(a_{1}\right)-\left(a_{3}\right)$, respectively. Shown are axis projections for ellipses whose centers lie on a square grid that surrounds the L point. These projections (short lines) are the tangents to the streamlines; visually, their overall pattern approximates the streamline pattern. The long straight lines are the separatixes calculated from Eq. (99), and as can be seen are in full accord with the patterns shown here, as well as with numerous other examples of these patterns that are not shown. ( $\left(b_{1}\right)$ This streamline pattern is similar to the contour pattern surrounding a saddle point. $\quad\left(b_{2}\right)$ These streamlines spiral around the L point. $\quad\left(b_{3}\right)$ Here the streamlines form right and left leaning parabolic-like curves "centered" on the right leaning separatix; the fields of right and left leaning "parabolas" are separated by the left leaning separatix. These three patterns are the only ones found in our simulation. We find that each pattern is tied to a particular combination of $I$ and $\Lambda$ : in all of the numerous cases tested for axes $\boldsymbol{\alpha}, \boldsymbol{\beta}$, and $\boldsymbol{\gamma}$, when $I=-1, \Lambda=4$, the pattern is qualitatively similar to that in $\left(\mathrm{b}_{1}\right)$; when $I=+1$ and $\Lambda=0$ the pattern is qualitatively similar to that in $\left(\mathrm{b}_{2}\right)$; and when $I=+1$ and $\Lambda=4$ the pattern is qualitatively similar to that in $\left(b_{3}\right) . \quad\left(c_{1}\right)-\left(c_{3}\right)$ Endpoint curves in $\pi_{0}$ corresponding to the axis projections in $\left(a_{1}\right)-\left(a_{3}\right)$. As can be seen, and as is expected (Fig. 3), $\Lambda$ equals the number of Z-axis crossings of the endpoint curve in $\pi_{0}$. This has been verified for all $10^{6}$ realizations in our random field database. 


\section{Theory}

The above results can be obtained analytically from the properties of the streamlines. These streamlines, expressible as the family of curves $y(x)$, are the solutions of

$$
\frac{d y}{d x}=\frac{V_{y}}{V_{x}}=\frac{F_{y x} x+F_{y y} y}{F_{x x} x+F_{x y} y}
$$

where in writing the second form we have used Eq. (3); $F_{x x}, F_{x y}$, etc., are given in Eq. (4).

Eq. (6) is a classic exercise in the theory of ordinary differential equations, and the properties of its solutions are well known [see for example 17]. There are six types of solution, three of which are unstable under small perturbation, and are therefore nongeneric and do not appear in our simulations, and three which are stable and which do appear. For completeness, and in order to help the reader avoid a possible misinterpretation of previous illustrations of $\boldsymbol{\beta}, \boldsymbol{\gamma}$ streamlines $[4,7,11]$, below we consider briefly all six types.

The solutions of Eq. (6) are characterized by both index $I$ and the discriminant

$$
\Delta=\left(F_{x x}-F_{y y}\right)^{2}+4 F_{x y} F_{y x}
$$

When $\Delta<0, \Lambda=0$, when $\Delta=0, \Lambda=2$, and when $\Delta>0, \Lambda=4$. As discussed below, the case $\Delta=0, \Lambda=2$ corresponds to one of the unstable solutions and is not seen in our simulation; the other two possibilities are.

The rule discussed above, that for $I_{i}=-1, \Lambda_{i} \equiv 4, i=\alpha, \beta, \gamma$, can be easily proven by rewriting Eq. (7) as

$$
\begin{aligned}
\Delta & =\left(F_{x x}+F_{y y}\right)^{2}-4 \mathcal{I}, \\
\mathcal{I} & =F_{x x} F_{y y}-F_{x y} F_{y x} .
\end{aligned}
$$

Recalling from Eq. (5) that $I=\operatorname{sign}(\mathcal{I})$, it immediately follows that if $I<0, \Delta>0$, and as a result, $\Lambda \equiv 4$.

The six solutions of Eq. (6) are illustrated in Fig. 9, where the three unstable (stable) solutions are labelled $\mathrm{U}_{1}, \mathrm{U}_{2}$, and $\mathrm{U}_{3}\left(\mathrm{~S}_{1}, \mathrm{~S}_{2}\right.$, and $\left.\mathrm{S}_{3}\right)$.

$\mathrm{U}_{1}$ corresponds to $d y / d x=y / x$, and requires $F_{x x}=F_{y y}$ and $F_{x y}=F_{y x}=0$. For these values of the parameters $\Delta=0$, and for this special case $\Lambda=\infty$ rather than $\Lambda=2$. When the stringent conditions on $F$ are no longer met, $\Delta \neq 0$, and $\mathrm{U}_{1}$ transforms into the stable configuration $\mathrm{S}_{2}\left(\mathrm{~S}_{3}\right)$, if $\Delta<0(\Delta>0)$, in both cases preserving index $I$, as expected.

$\mathrm{U}_{2}$ corresponds to the condition $F_{y y}=-F_{x x}$. When this special condition is met the streamlines form conic sections: ellipses if $\Delta<0$; hyperbolas if $\Delta>0$. These hyperbolas are organized into the contour pattern of a saddle point. When the special condition on $F$ is no longer met the ellipses open into the stable spiral $\mathrm{S}_{2}$, whereas the pattern of the hyperbolas is maintained as the stable solution $\mathrm{S}_{1}$, although the curves themselves are no longer hyperbolas. As expected, in both cases index $I$ is conserved during the transformation.

$\mathrm{U}_{3}$ corresponds to the special condition $F_{x y} F_{y x}=-\frac{1}{4}\left(F_{x x}-F_{y y}\right)^{2}$. When this special condition is met $\Delta=0$ and $\Lambda=2$. When the condition on the $F^{\prime} s$ is no longer met $\mathrm{U}_{3}$ transforms into the stable solution $\mathrm{S}_{2}\left(\mathrm{~S}_{3}\right)$ if $\Delta<0(\Delta>0)$; again, index $I$ is conserved during the transformation.

Worth noting is that when the special conditions on $F$ are very nearly satisfied, as can and does happen in a random (or other) field, the pattern of streamlines can closely approximate the 
unstable solutions, possibly resulting in the misleading impression that these solutions have been observed, and are therefore stable.

For the three stable solutions $\mathrm{S}_{1}, \mathrm{~S}_{2}$, and $\mathrm{S}_{3}$, indices $I$ and $\Lambda$ are the following: for $\mathrm{S}_{1}, I=-1$ and $\Lambda=4$; for $\mathrm{S}_{2}, I=+1$ and $\Lambda=0$; for $\mathrm{S}_{3}, I=+1$ and $\Lambda=4$. These index combinations uniquely determine the streamline pattern, and parallel in some sense the three index combinations for the three types of stable C points, "star", "lemon", and "monstar" [4,11-13,16], although the patterns themselves differ for the two cases. The $\mathrm{C}$ point combinations are: for the star, $I=-1$ and $\Lambda=3$; for the lemon, $I=+1$ and $\Lambda=1$; for the monstar, $I=+1$ and $\Lambda=3$.

When $\Lambda=4$, the straight-line streamlines passing through the origin (L point) are given by

$$
y_{ \pm}=\frac{F_{y y}-F_{x x} \pm \sqrt{\Delta}}{2 F_{x y}} x .
$$

These lines are separatixes that divide the field into four quadrants in which the sign of the curvature of the streamlines changes across a separatix.

We find that our simulations (Fig. 8) are in full agreement with all of the above, and that all three stable solutions $\mathrm{S}_{1}, \mathrm{~S}_{2}$, and $\mathrm{S}_{3}$, are present for each axis $\boldsymbol{\alpha}, \boldsymbol{\beta}$, and $\boldsymbol{\gamma}$, and for each type of structure $-\alpha$-cones, r-rings, and Möbius strips. This reflects the fact that for each of these structures all three index combinations, $I=-1, \Lambda=4\left(\mathrm{~S}_{1}\right), I=1, \Lambda=0\left(\mathrm{~S}_{2}\right)$, and $I=1, \Lambda=4$ $\left(\mathrm{S}_{3}\right)$, appear.
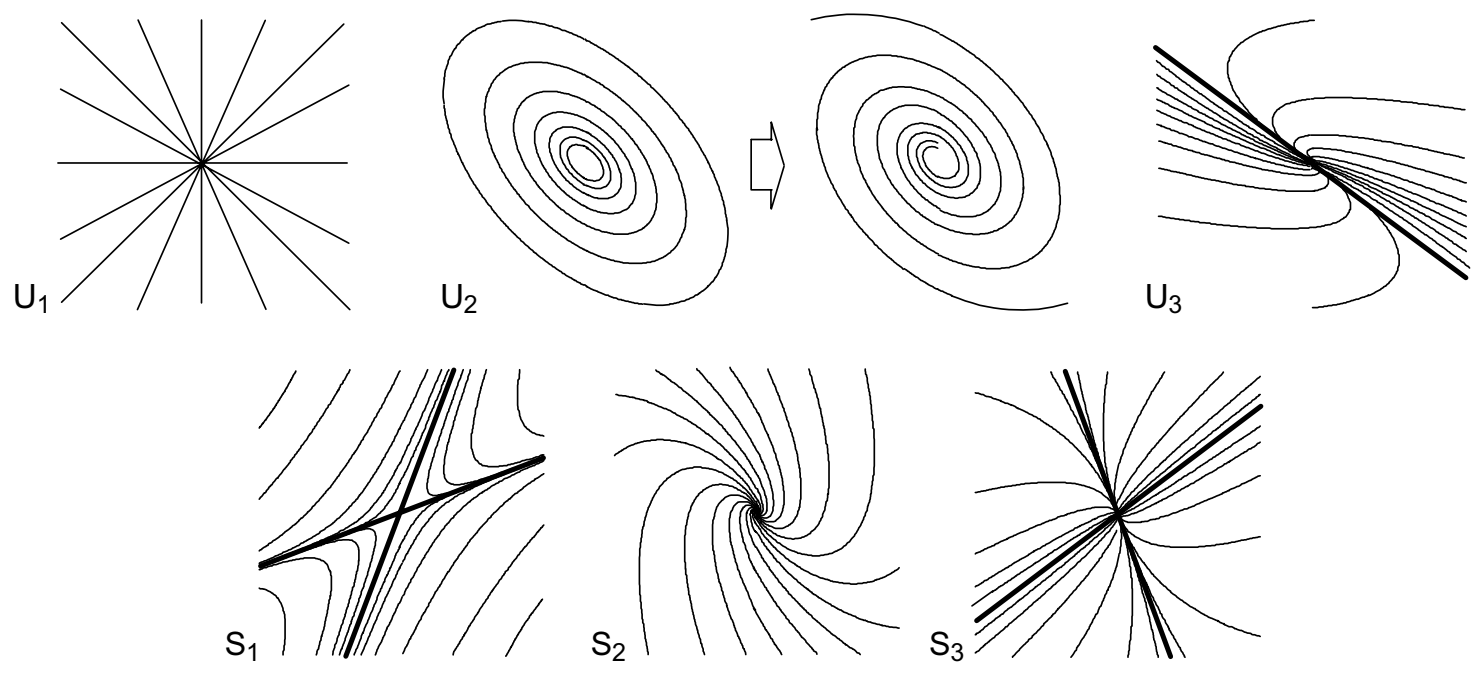

FIG. 9: Solutions of Eq. (6). $\mathrm{U}_{1}-\mathrm{U}_{3}\left(\mathrm{~S}_{1}-\mathrm{S}_{3}\right)$ are unstable (stable) solutions. The parameters used in plotting these solutions are for the unstable solutions: $\mathrm{U}_{1}, F_{x x}=1, F_{x y}=F_{y x}=0, F_{y y}=1 ; \mathrm{U}_{2}$, $F_{x x}=1 / 2-\delta(\delta=0 \Rightarrow \delta=0.1), F_{x y}=1, F_{y x}=-1, F_{y y}=-1 / 2 ; \mathrm{U}_{3}, F_{x x}=1, F_{x y}=1, F_{y x}=$ $-1 / 4, F_{y y}=0$. For the stable solutions the parameters are: $\mathrm{S}_{1}, F_{x x}=1, F_{x y}=-1, F_{y x}=1, F_{y y}=-2 ; \mathrm{S}_{2}$, $F_{x x}=1, F_{x y}=-2, F_{y x}=3, F_{y y}=4 ; \mathrm{S}_{3}, F_{x x}=3, F_{x y}=1 / 2, F_{y x}=1, F_{y y}=2 . \quad$ These parameters were chosen to yield plots that approximately match the streamlines in Fig. 8. $\mathrm{S}_{1}$ approximately corresponds to the pattern in Fig. $8 \mathrm{~b}_{1} ; \mathrm{S}_{2}$ to the pattern in Fig. $8 \mathrm{~b}_{2} ; \mathrm{S}_{3}$ to the pattern in Fig. $8 \mathrm{~b}_{3}$.

\section{Index Summary}

We briefly summarize here the major properties of the 15 indices that characterize the Möbius strips, r-rings, and $\alpha$-cones that surround L lines. 
a. $I_{\alpha}, I_{\beta, \gamma}, \Lambda_{\alpha}, \Lambda_{\beta}, \Lambda_{\gamma}$. These five indices characterize the projections of axes $\boldsymbol{\alpha}, \boldsymbol{\beta}$, and $\boldsymbol{\gamma}$ onto the principal plane $\Sigma_{0}$. Index $I$ measures the winding number of these projections, $\Lambda$ counts the number of straight streamlines (separatixes) that terminate (or originate) on the L point. For all three axes $I$ takes the values \pm 1 and $\Lambda$ takes the values 0,4 . If $I=-1, \Lambda \equiv 4$, whereas for $I=+1, \Lambda=0,4$. Each of the three possible combinations of $I$ and $\Lambda$ defines a unique streamline pattern, Figs. 8 and 9 .

b. $d \tau_{\alpha}, \tau_{\beta}, d \tau_{\beta}, \tau_{\gamma}, d \tau_{\gamma}$. These five indices characterize the projections of axes $\boldsymbol{\alpha}$, $\boldsymbol{\beta}$, and $\gamma$ onto the plane $\tau_{0}$, Fig. 3. $\tau$ measures the winding number of the axes about the circle of ellipse centers, $\sigma_{0}$. For Möbius strips $\tau= \pm 2$, where $+2(-2)$ implies a left-handed (right-handed) two-turn helix, Figs. 1 and 4 . For r-rings (and $\alpha$-cones) $\tau=0$.

$d \tau$ measures the winding number of the tangent to the endpoint curve. If this is a simple closed curve $d \tau=+2(d \tau=-2)$, Fig. 4 and 6. If the endpoint curve generates a figure of eight, which can occur for axes $\boldsymbol{\beta}$ and $\boldsymbol{\gamma}$, but not for axis $\boldsymbol{\alpha}, d \tau=0$, Fig. 5. When $\tau= \pm 2, d \tau=\tau$.

c. $d \pi_{\alpha}, \pi_{\beta}, d \pi_{\beta}, \pi_{\gamma}, d \pi_{\gamma}$. These five indices characterize the projections of axes $\boldsymbol{\alpha}, \boldsymbol{\beta}$, and $\boldsymbol{\gamma}$ onto the plane $\pi_{0}$, Fig. 3. If the endpoint curve in $\pi_{0}$ encloses the origin (circle of ellipse centers), which can occur for Möbius strips and r-rings, but not for $\alpha$-cones, $\pi= \pm 2$, otherwise $\pi=0$. $d \pi$ measures the rotation of the tangent to the $\pi_{0}$ endpoint curve. The possible values of this index for the different axes are the same as those for $d \tau$. When $\pi= \pm 2, d \pi=\pi$. $N_{Z}$, the number of times the endpoint curve in $\pi_{0}$ crosses the $Z$-axis, is equal to $\Lambda$, the line classification index, so $N_{Z}$ could replace $\Lambda$; no index or combination of indices in $\pi_{0}$ and/or $\tau_{0}$, however, can replace $I$.

For a given structure - Möbius strip, r-ring, or $\alpha$-cone - indices $\tau$ and $d \tau$ of $\tau_{0}$, and indices $\pi$ and $d \pi$ of $\pi_{0}$, can be, and often are, different, and to a large extent these indices are also independent of the indices $I$ and $\Lambda$ that describe the projection onto $\Sigma_{0}$. There are, however, index combinations that do not occur; in the following section these missing combinations are summarized in the form of selection rules.

\section{STATISTICS}

We discuss here the probabilities of various combinations of the 15 indices that characterize the Möbius strips, r-rings, and $\alpha$-cones that surround L lines, starting with those combinations for which the probability is zero. These zero probability combinations are summarized below in the form of binary and ternary selection rules. Quaternary and higher-order rules may also exist. Discussed below are simple, systematic methods for identifying all true binary and ternary rules; in contrast, there do not appear to be equivalent methods for identifying true higher-order rules (i.e. rules that do not incorporate combinations of lower-order rules).

\section{A. Selection Rules}

The binary and ternary selection rules listed below were either derived from results discussed earlier, or deduced from the absence of various configurations in our $10^{6}$ entry database. These latter, empirical rules await confirmation from theory.

Finding the empirical rules was facilitated by the use of $2 \mathrm{D}$ and $3 \mathrm{D}$ correlation (scatter) plots. Of the 105 2D plots of two index combinations, 17 had missing configurations that led to the binary selection rules listed below. The number of 3D, three-index plots was 455; of these 198 had missing configurations, most of which, however, involved combinations of missing two-index configurations already included in the binary rules. The ternary selection rules listed below were obtained from the 12 plots that contained true three-index missing configurations (some plots yielded more than 
one rule). Typical examples of $2 \mathrm{D}$ and $3 \mathrm{D}$ correlation plots with missing configurations are shown in Fig. 10.
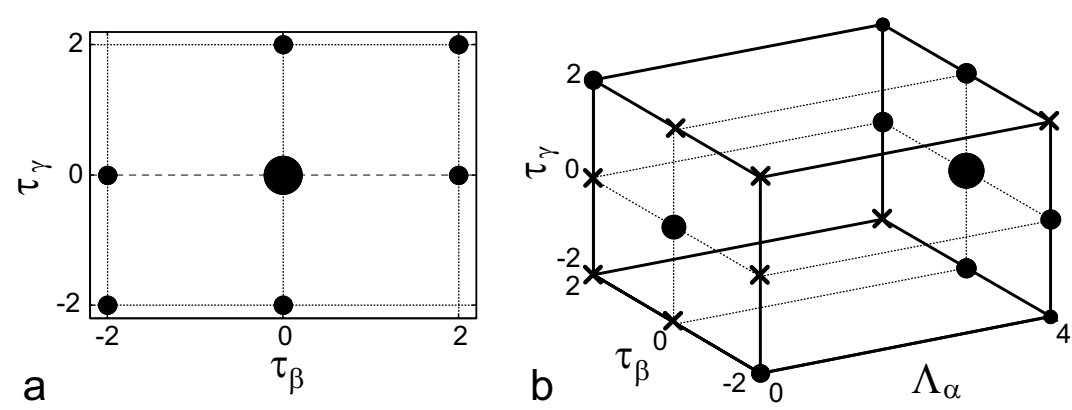

FIG. 10: Index correlation plots. The relative areas of the circles approximate the relative probabilities of the different configurations. (a) $2 \mathrm{D}$ plot of the index combination $\tau_{\beta}-\tau_{\gamma}$. Empty grid points correspond to missing configurations. (b) 3D plot of the index combination $\Lambda_{\alpha}-\tau_{\beta}-\tau_{\gamma}$. Missing configurations are marked by $\times$ 's. The reader may enjoy identifying the selection rules obtained from these plots.

The binary (ternary) selections rules acting alone reduce the number of configurations of all 15 indices from 839, 808 to $16,344(98,864)$; both sets of rules acting together yield 8, 248 allowed configurations, of these 5,562 have been observed in our simulations.

\section{Binary rules}

These rules involve pairs of axes. They state that the following two-index configurations are forbidden:

$$
\begin{aligned}
I_{i} & =-1 \quad \& \quad \Lambda_{i}=0, i=\alpha, \beta, \gamma . \\
\left|\pi_{i}\right| & =2 \quad \& \quad \Lambda_{i}=0, i=\beta, \gamma . \\
\left|\tau_{i}\right| & =2 \& \Lambda_{j}=0, i j=\beta \gamma, \gamma \beta . \\
d \tau_{i} & =0 \quad \& \quad \Lambda_{i}=0, i=\beta, \gamma . \\
d \pi_{i} & =0 \& \Lambda_{j}=0, i j=\beta \gamma, \gamma \beta . \\
\left|\tau_{i}\right| & =2 \& d \tau_{i} \neq \tau_{i}, i=\beta, \gamma . \\
\left|\pi_{i}\right| & =2 \& d \pi_{i} \neq \pi_{i}, i=\beta, \gamma . \\
\left|\tau_{\beta}\right| & =2 \&\left|\tau_{\gamma}\right|=2 \& \operatorname{sign}\left(\tau_{\beta}\right) \neq \operatorname{sign}\left(\tau_{\gamma}\right) . \\
\left|\pi_{\beta}\right| & =2 \&\left|\pi_{\gamma}\right|=2 \& \operatorname{sign}\left(\pi_{\beta}\right) \neq \operatorname{sign}\left(\pi_{\gamma}\right) .
\end{aligned}
$$

The above rules either follow from already discussed, or imply new, geometrical connections between the structures formed by the different axes for a given set of wavefield parameters. Below we briefly discuss these connections.

Rule 2.1. This rule is proven in Eq. (8).

Rule 2.2. This rule follows from an already discussed geometrical connection: $|\pi|=2$ requires the endpoint curve in $\pi_{0}$ to cross the $Z$-axis four times, Section III.B.2a and Fig. 44 at each such crossing the axis projection is radial, Fig. 3, and so $\Lambda=4$.

Rule 2.3. This rule follows from a an already discussed geometrical connection: $|\tau|=2$ (i.e. a Möbius strip) requires the endpoint curve in $\tau_{0}$ to cross the $Z$-axis four times; at each such crossing the axis projection is tangential, Fig. 3. Because, as already noted in Section III.A, the 
projections of axes $\boldsymbol{\beta}$ and $\boldsymbol{\gamma}$ onto $\Sigma_{0}$ are orthogonal, where the $\boldsymbol{\beta}(\boldsymbol{\gamma})$ projection is tangential the $\gamma(\boldsymbol{\beta})$ projection is radial, and so $\Lambda_{\gamma}=4\left(\Lambda_{\beta}=4\right)$.

Rule 2.4. This rule implies a new geometrical connection: if the endpoint curve in $\tau_{0}$ is a figure of eight, it must cross the $Z$-axis four times (see the explanation of Rule 2.3).

Rule 2.5. This rule implies a new geometrical connection: if the endpoint curve of axis $\boldsymbol{\beta}(\boldsymbol{\gamma})$ in $\pi_{0}$ is a figure of eight, the endpoint curve of axis $\boldsymbol{\gamma}(\boldsymbol{\beta})$ in $\pi_{0}$ must cross the $Z$-axis four times.

Rules 2.6 and 2.7. These rules follows from already discussed geometrical connections: if $|\tau|=2(|\pi|=2)$ the endpoint curve is elliptical in shape, and therefore $d \tau=\tau(d \pi=\pi)$, Fig. 4 .

Rule 2.8. This rule implies a new geometrical connection: if both axis $\boldsymbol{\beta}$ and axis $\boldsymbol{\gamma}$ generate Möbius strips, both strips must have the same handedness.

Rule 2.9. This rule implies a new geometrical connection: if the endpoint curves in $\pi_{0}$ of axis $\boldsymbol{\beta}$ and axis $\boldsymbol{\gamma}$ are both elliptical in shape, both curves must have the same sign.

\section{Ternary Rules}

These rules involve three different axes. They state that the following three-index configurations are forbidden:

$$
\begin{aligned}
\Lambda_{\alpha} & =0 \& I_{\beta, \gamma}=-1 \quad \& \quad \tau_{i}=0, i=\beta, \gamma . \\
\Lambda_{\alpha} & =0 \& I_{\beta, \gamma}=-1 \& d \tau_{i}=0, i=\beta, \gamma . \\
\Lambda_{\alpha} & =0 \& \Lambda_{i}=0 \&\left|\tau_{i}\right|=2, i=\beta, \gamma . \\
\Lambda_{\alpha} & =0 \&\left|\tau_{i}\right|=2 \& \tau_{j}=0, i j=\beta \gamma, \gamma \beta . \\
\Lambda_{\alpha} & =0 \&\left|\tau_{i}\right|=2 \& d \tau_{j} \neq \tau_{i}, i j=\beta \gamma, \gamma \beta . \\
\Lambda_{\alpha} & =0 \&\left|\tau_{i}\right|=2 \& d \tau_{\alpha} \neq \tau_{i}, i=\beta, \gamma . \\
\left|\tau_{\beta}\right| & =2 \& \tau_{\gamma}=\tau_{\beta} \& d \tau_{\alpha} \neq \tau_{\beta} . \\
\left|\pi_{\beta}\right| & =2 \& \pi_{\gamma}=\pi_{\beta} \& d \pi_{\alpha} \neq \pi_{\beta} . \\
\left|\tau_{i}\right| & =2 \& d \tau_{\alpha}=-\tau_{j}, i j=\beta \gamma, \gamma \beta . \\
\left|\pi_{i}\right| & =2 \& d \pi_{\alpha}=-\pi_{j}, i j=\beta \gamma, \gamma \beta . \\
\left|\tau_{i}\right| & =2 \& d \tau_{\alpha}=-d \tau_{j}, i j=\beta \gamma, \gamma \beta . \\
\left|\pi_{i}\right| & =2 \& d \pi_{\alpha}=-d \pi_{j}, i j=\beta \gamma, \gamma \beta .
\end{aligned}
$$

All the ternary rules involve connections between $\alpha$-cones and Mobius strips or r-rings. These connections, all of which are new, appear difficult to fathom in easily understood, simple geometrical terms. For example, Rule 3.1 states that if the streamlines of axis $\boldsymbol{\alpha}$ form a spiral, Fig. 9.S2, and those of axis $\boldsymbol{\beta}$ (axis $\boldsymbol{\gamma}$ ) form a saddle point, Fig. 9-S1, then axis $\boldsymbol{\beta}$ (axis $\boldsymbol{\gamma}$ ) itself must generate a Möbius strip.

\section{B. Probabilities}

The relative probabilities of all 15-index combinations found in our simulation are displayed in Fig. 11 in the form of a modified Zipf plot. In this plot the number of occurrences of a configuration, $N$, is plotted vs. the rank of the configuration, $R$, where configurations are ranked in descending order of probability. As can be seen, for $R>1, N$ decreases step-wise to one with increasing rank in a quasi-continuous fashion. 
But if $N$ decreases quasi-continuously to one, then what meaning can be attached to the empirical selection rules for which $N=0$ ? The answer is that there is a qualitative difference between the two-index (three-index) and the fifteen-index cases, because in the case of the two-index, binary (three-index, ternary), selection rules, the number of observed occurrences is either greater than $10^{5}\left(10^{4}\right)$, or zero.

Shown in the inset of Fig. 11 is the number of unique index combinations, $n$, as a function of the number of realizations, $r$, where $r$ is incremented sequentially in steps of $10^{5}$. As can be seen, $n$ appears to asymptote to the power law $n=2,244 r^{0.0657}$. The parameters in this law were chosen such that for $r=10^{6}$, i.e. for our simulation, $n=5562$, i.e. the number actually observed. The binary and ternary selection rules yield 8,248 possible configurations. Assuming that higher-order rules do not significantly reduce this number, and that the power law remains unchanged for much larger $r$, some $4 \times 10^{8}$ realizations would be needed to capture all possibilities. More realistically, the yield of new configurations can be expected to fall off with increasing $r$, so that even $10^{9}$ realizations might not suffice to yield all allowed configurations.

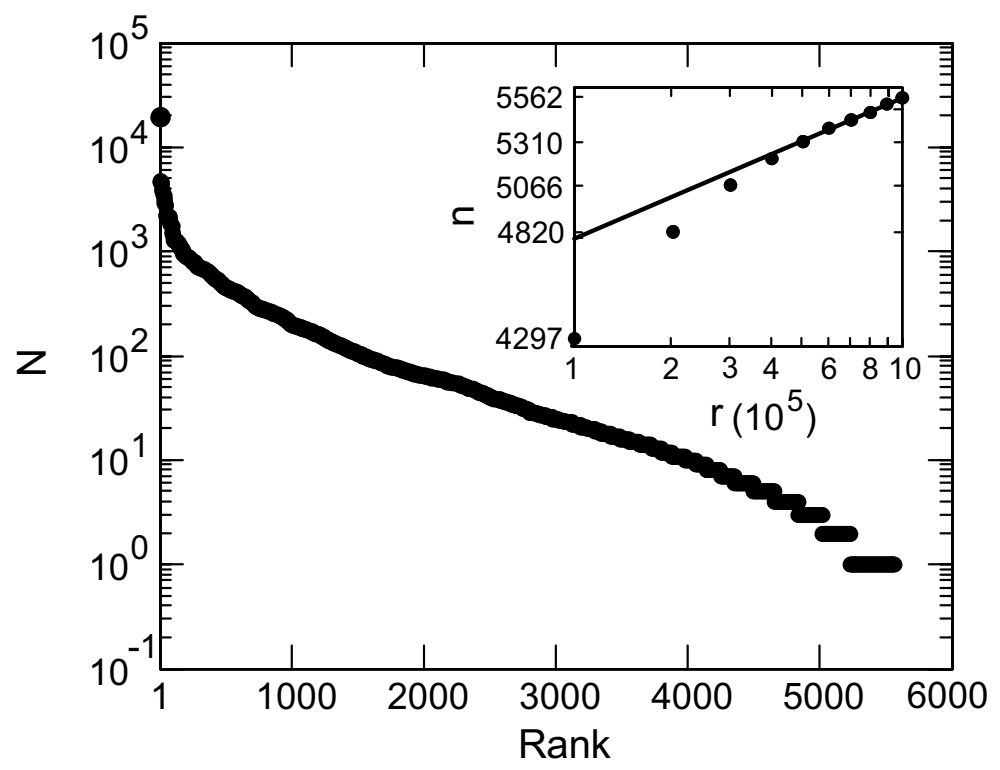

FIG. 11: Modified Zipf plot. Shown is the number of configurations $N$ observed in our simulation vs. configuration rank $R$. The inset shows a log-log plot of the number of unique configurations, $n$, as a function of the number of realizations $r$. The straight line asymptote is the power law $n=2,244 r^{0.0657}$. In the four most probable configurations (large dot at Rank $=1$ ), which all have substantially the same probability, both axes $\boldsymbol{\beta}$ and $\boldsymbol{\gamma}$ form Möbius strips $\left(\left|\tau_{\beta}\right|=\left|\tau_{\gamma}\right|==2\right)$, the streamlines of axis $\boldsymbol{\alpha}$ form a spiral $\left(\Lambda_{\alpha}=0\right)$, and those of both axis $\boldsymbol{\beta}$ and axis $\gamma$ form saddle points $\left(I_{\alpha, \beta}=-1, \Lambda_{\beta}=\Lambda_{\gamma}=4\right)$.

Listed below are the probabilities of occurrence in our database of a number of configurations that may be of special interest. These probabilities are not densities because they do not include the appropriate Jacobian. Berry and Dennis have presented a Jacobian appropriate for some 3D L line properties [12], but this Jacobian does not appear to be suitable for Möbius strips, r-rings, and $\alpha$-cones in $\Sigma_{0}$. The reason is that these structures and their statistics can change importantly when the plane of observation $\Sigma$ is tilted away from $\Sigma_{0}$ by a small but finite, amount, a property not shared by the Jacobian in [12]. Because the probabilities listed below are not densities they are not easily measured experimentally; they are, however, amenable to calculation. Fig. 12 presents some examples of transformations of Möbius strips, r-rings, and $\alpha$-cones under tilt of the plane of observation; these transformed structures differ importantly from those appearing in $\Sigma_{0}$. A more complete discussion of transformations under tilt will be presented elsewhere. 

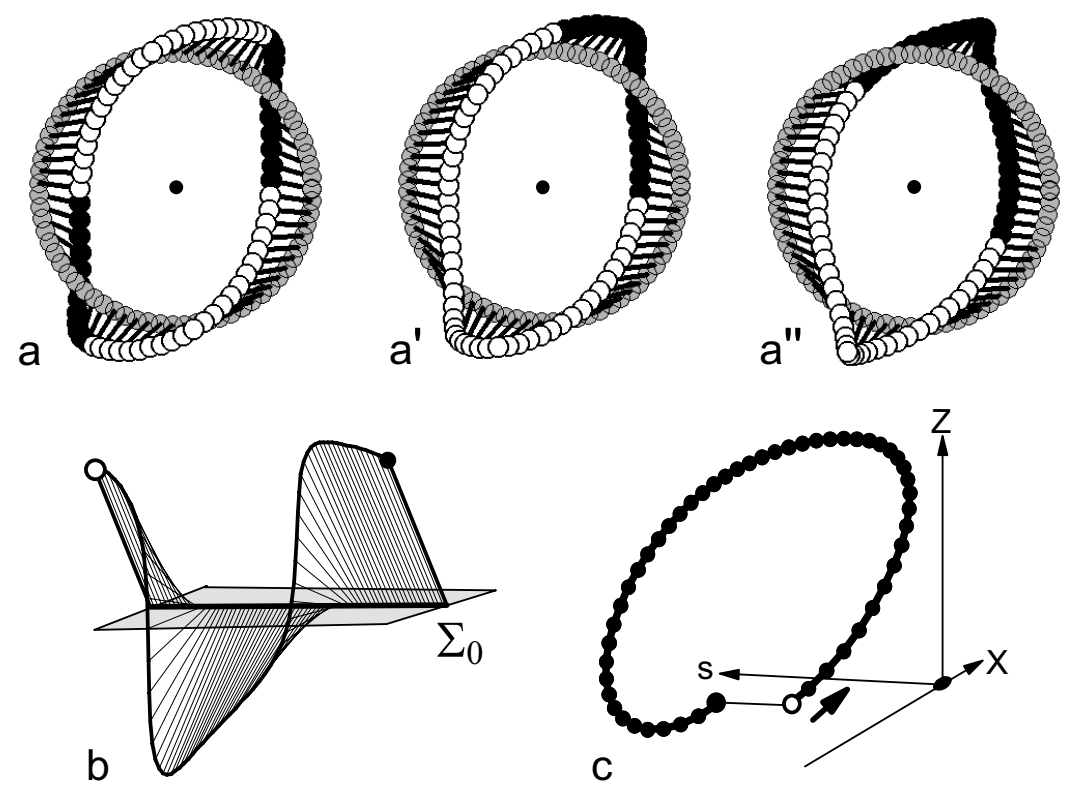

FIG. 12: Transformations of Möbius strips, r-rings, and $\alpha$-cones. As the plane of observation $\Sigma$ is tilted away from $\Sigma_{0}$, different sets of ellipses occupy the surrounding circle. The structures, indices, and statistics of the Möbius strips, r-rings, and $\alpha$-cones generated by these ellipses can change importantly with tilt angle. For reasons given below, in the examples shown here the radius $r$ of the surrounding circle is $r=0.1$, instead of the value used throughout this report, $r=10^{-4}$; in all the cases shown, when the tilt angle is zero, the Möbius strips, r-rings, and $\alpha$-cones are, except for a scale factor, virtually identical for $r=0.1$ and $r=10^{-4}$. (a) The Möbius strip in Fig. 1 p for zero tilt angle, i.e. $\Sigma=\Sigma_{0}$. As in Fig. 1 p, axis endpoints are shown by small black (white) circles if the endpoint lies above (below) the circle of ellipse centers (small gray circles). As can be seen, just like in Fig. 1 $1 \mathrm{~b}$ the endpoints and the ellipse centers form interlocking rings with four crossings, and $\tau=+2$. (a') The strip in (a) for $\Sigma$ tilted away from $\Sigma_{0}$ by $5^{\circ}$. Here the rings still interlock, but with only two crossings instead of four, and $\tau=+1$ instead of $\tau=+2$. (a") The strip in (a) for $\Sigma$ tilted away from $\Sigma_{0}$ by $10^{\circ}$. Here the rings no longer interlock, and $\tau=0$. (b) The opened r-ring in Fig. $1 \mathrm{~d}$ ' for a tilt angle of $5^{\circ}$. Here the axes oscillate through only one sinusoidal cycle instead of through two, and $d \tau=+1$ instead of +2 as in Fig. 5 ( (c) The $\alpha$-cone in Figs. 1 and 6 for a $5^{\circ}$ tilt angle. Here the endpoint spiral has only one turn instead of two, and $d \tau=+1$ instead of +2 as in Fig. 6r. The reason for using $r=0.1$ instead of $r=10^{-4}$ is that the tilt angle required for a given transformation decreases with $r$, becoming so small for $r=10^{-4}$ that experimentation becomes impractical. For $r_{0}=0.1$, however, the tilt angle is several degrees, and we use the larger value of $r$ to demonstrate that the transformations described here can be accessible to experiment.

From our simulation we find the following:

(i) As expected, positive and negative values for all indices appear with equal probabilities.

(ii) The fraction of $\mathrm{L}$ lines or $\mathrm{L}$ line segments surrounded by Möbius strips is 0.359 , the remaining lines are surrounded by r-rings. This fraction holds for both axis $\boldsymbol{\beta}$ and axis $\boldsymbol{\gamma}$ strips.

(iii) The fraction of $L$ lines or $L$ line segments in which both axis $\boldsymbol{\beta}$ and axis $\boldsymbol{\gamma}$ of the surrounding ellipses generate Möbius strips (r-rings) is 0.185 (0.468).

(iv) The fraction of Möbius strips whose $\pi_{0}$ endpoint curves form simple closed curves is 0.815 , the remaining endpoint curves are figures of eight. This fraction holds for both axis $\boldsymbol{\beta}$ and axis $\boldsymbol{\gamma}$.

$(v)$ The fraction of r-rings whose $\tau_{0}$ or $\pi_{0}$ endpoint curves form simple closed curves is 0.718 , the remaining endpoint curves form figures of eight. This fraction holds for both axes $\boldsymbol{\beta}$ and $\boldsymbol{\gamma}$.

(vi) The fraction of axis $\boldsymbol{\beta}$ Mobius strips (r-rings) with $I_{\beta, \gamma}=+1$ and $\Lambda_{\beta}=0$ is 0.808 (0.547), for the remaining strips (rings) with $I_{\beta, \gamma}=+1, \Lambda_{\beta}=4$. The same fractions with subscripts $\beta$ and $\gamma$ interchanged hold for axis $\gamma$ Mobius strips (r-rings). 
(vii) The fraction of Mobius strips (r-rings) with $\Lambda_{\alpha}=0$ is 0.408 (0.228), for the remaining strips (rings), $\Lambda_{\alpha}=4$. These fractions hold for both axis $\boldsymbol{\beta}$ and axis $\boldsymbol{\gamma}$ Mobius strips (r-rings).

(vii) The fraction of $\alpha$-cones with $I_{\alpha}=+1$ and $\Lambda_{\alpha}=0$ is 0.586 , for the remaining $I_{\alpha}=+1$ cones $\Lambda_{\alpha}=4$.

Although there are an enormous number of other statistical combinations that are easily obtained from our database, we believe that the above are likely to be of the greatest interest since they relate to the major geometric properties of, and interconnections between, the different structures.

\section{SUMMARY}

The axes of the ellipses surrounding lines of linear polarization, L lines, in a 3D optical ellipse field have been shown to generate three distinctly different types of structures. In a plane normal to the direction of polarization of an L point on an L line the major and minor axes of the surrounding ellipses that lie on small circles surrounding the point generate Möbius strips or r-rings (rippled rings), whereas the major axes of these ellipses generate cones ( $\alpha$-cones). The Möbius strips have two whole twists and can be either right- or left-handed screws. These three different types of structures were characterized by 15 indices: 12 winding numbers that characterize the projections of the different structures onto three orthogonal planes, $\Sigma_{0}, \tau_{0}$, and $\pi_{0}$, and three indices that characterize the streamlines formed by the projections onto $\Sigma_{0}$. Analytical (numerical) results were given for all indices in $\Sigma_{0}$ (in $\tau_{0}$ and in $\pi_{0}$ ). Binary and ternary selection rules were presented that reduce the number of possible configurations from 839, 808 to 8,248, of which 5, 562 have been observed in a computer simulation; probabilities of occurrence were given for the most important of these configurations.

Experimental measurement of the various structures require determination of all three orthogonal components of the optical field; such measurements are currently feasible both in the microwave $[9,10,18,19]$, and in the optical $[20-27]$, regions of the spectrum.

\section{References}

[1] I. Freund, "Cones, spirals, and Mobius strips in elliptically polarized light," Opt. Commun. 249, 7-22 (2005).

[2] I. Freund, "Hidden order in optical ellipse fields: I. Ordinary ellipses," Opt. Commun. 256, 220-241 (2005).

[3] I. Freund, "Optical Mobius strips in three-dimensional ellipse fields: Lines of circular polarization," Opt. Commun., submitted.

[4] J. F. Nye, Natural Focusing and Fine Structure of Light (IOP Publ., Bristol, 1999).

[5] J. F. Nye, "Polarization effects in the diffraction of electromagnetic waves: the role of disclinations," Proc. Roy. Soc. Lond. A 387, 105-132 (1983).

[6] J. F. Nye, "Lines of circular polarization in electromagnetic wave fields," Proc. Roy. Soc. Lond. A 389, 279-290 (1983).

[7] J. F. Nye and J. V. Hajnal, "The wave structure of monochromatic electromagnetic radiation," Proc. Roy. Soc. Lond. A 409, 21-36 (1987).

[8] J. V. Hajnal, "Singularities in the transverse fields of electromagnetic waves. I. Theory," Proc. Roy. Soc. Lond. A 414, 433-446 (1987).

[9] J. V. Hajnal, "Singularities in the transverse fields of electromagnetic waves. II Observations on the electric field," Proc. Roy. Soc. Lond. A 414, 447-468 (1987). 
[10] J. V. Hajnal, "Observations of singularities in the electric and magnetic fields of freely propagating microwaves," Proc. Roy. Soc. Lond. A 430, 413-421 (1990).

[11]. M. V. Berry, "Geometry of phase and polarization singularities, illustrated by edge diffraction and the tides," in Second International Conference on Singular Optics, M. S. Soskin and M. V. Vasnetsov Eds., Proc. SPIE 4403, 1-12 (2001).

[12] M. V. Berry and M. R. Dennis, "Polarization singularities in isotropic random vector waves," Proc. Roy. Soc. Lond. A 457, 141-155 (2001).

[13] M. V. Berry, "Index formulae for singular lines of polarization," J. Opt. A 6, 675-678 (20044.

[14] M. Born and E. W. Wolf, Principles of Optics (Pergamon Press, Oxford, 1959).

[15] I. Freund, 'Coherency matrix description of optical polarization singularities,' J. Opt. A 6, S229-S234 (2004).

[16] M. V. Berry and J. H. Hannay, "Umbilic points on Gaussian random surfaces," J. Phys. A 10, 1809-1821 (1977).

[17] L. R. Ford, Differential Equations (McGraw-Hill, New York, 1955)

[18] S. Zhang and A. Z. Genack, "Statistics of Diffusive and Localized Fields in the Vortex Core," Phys. Rev. Lett. 99, 203901 (2007).

[19] S. Zhang, B. Hu, P. Sebbah, and A. Z. Genack, "Speckle Evolution of Diffusive and Localized Waves," Phys. Rev. Lett. 99, 063902 (2007).

[20] R. Dandliker, I. Marki, M. Salt, and A. Nesci, "Measuring optical phase singularities at subwavelength resolution," J. Optics A 6, S189-S196 (2004).

[21] P. Tortora, R. Dandliker, W. Nakagawa, and L. Vaccaro, "Detection of non-paraxial optical fields by optical fiber tip probes," Opt. Commun. 259, 876-882 (2006).

[22] C. Rockstuhl, I. Marki, T. Scharf, M. Salt, H. P. Herzig, and R. Dandliker, "High resolution interference microscopy: A tool for probing optical waves in the far-field on a nanometric length scale," Current Nanoscience 2, 337-350 (2006).

[23] P. Tortora, E. Descrovi, L. Aeschimann, L. Vaccaro, H. P. Herzig, and R. Dandliker, "Selective coupling of HE11 and TM01 modes into microfabricated fully metal-coated quartz probes," Ultramicroscopy 107, 158-165 (2007).

[24] K. G. Lee, H. W. Kihm, J. E. Kihm, W. J. Choi, H. Kim, C. Ropers, D. J. Park, Y. C. Yoon, S. B. Choi, H. Woo, J. Kim, B. Lee, Q. H. Park, C. Lienau C, and D. S. Kim, "Vector field microscopic imaging of light," Nature Photonics 1, 53-56 (2007).

[25] Z. H. Kim and S. R. Leone, "Polarization-selective mapping of near-field intensity and phase around gold nanoparticles using apertureless near-field microscopy," Opt. Express 16, 1733-1741 (2008).

[26] M. Burresi, R. J. Engelen, A. Opheij, D. van Oosten, D. Mori, T. Baba, and L. Kuipers, "Observation of Polarization Singularities at the Nanoscale," Phys. Rev. Lett. 102, 033902 (2009).

[27] R. J. Engelen, D. Mori, T. Baba, and L. Kuipers, "Subwavelength Structure of the Evanescent Field of an Optical Bloch Wave," Phys. Rev. Lett. 102, 023902 (2009); Erratum: ibid. 049904 (2009). 